\title{
THE EFFECT OF ARQ BLOCK SIZE ON THE EFFICIENCY OF A WIRELESS ACCESS LINK USING ADAPTIVE MODULATION
}

\author{
Fraser Cameron and Moshe Zukerman \\ Department of Electrical and Electronic Engineering \\ The University of Melbourne, Parkville, Victoria 3052, Australia \\ Email:fkc@ee.mu.oz.au
}

\begin{abstract}
This paper optimizes a comprehensive set of parameters with the aim to minimize the bandwidth required for wireless transmission on a single link investigating the effect of ARQ blocksize on the adaptive link. The paper takes into consideration a comprehensive set of coding, modulation and teletraffic issues including the effects of retransmissions of erroneous packets using ARQ; modulation efficiency; FEC redundancy; and effect of traffic burstiness on loss and delay. Simulations are used to derive BER values for a range of modulation efficiency and FEC Code Rate parameters. These parameters are optimized to obtain maximal efficiency when looking at TCP over wireless multi-service media. We show that an adaptive system provides a relatively insensitive response to variation in transmitted block size with advantages.
\end{abstract}

Keywords: Wireless networks, FEC, ARQ, Adaptive modulation

\section{INTRODUCTION}

Personal and terminal mobility requires better area coverage, improved signal quality and reliability as well as the ability to interface with wired multimedia networks based on TCP/IP and/or ATM. According to current research and developments trends, the future Internet will support provision of specified Quality of Service (QoS) to customers which can be measured and guaranteed [6]. Unlike wired optical fibre networks where QoS requirements are mainly related to cell or packet loss probability due to congestion, in wireless broadband networks we must also consider access delay as an important QoS requirement affecting network dimensioning, and traffic control mechanisms.

To overcome error in a transparent mode of transmission, it is possible to use Forward Error Correction (FEC) and/or to reduce the number of symbols in the transmitted constellation in order to improve BER. Both of these 
solutions result in decreased channel capacity. In [2] and [3] it was shown that adaptive modulation is a powerful tool for performance optimisation the size of the constellation is an essential part of data services in wireless networks and the choice of that constellation has a high impact on the BER. In this paper it will be shown that adaptive modulation is a powerful tool in reducing wireless link sensitivity to ARQ block size which is an important result that leads to increased utilisation of the wireless link.

The basic idea behind an adaptive modulation scheme is to adjust the method of transmission to ensure that the maximum efficiency is achieved over the link at all time. For comments on implementability of adaptive modulation and the use of this research to GSM and other mobile networks see [3]. In general, a modulator accepts $M$ binary digits at a time and transmits a single symbol. This provides Modulation Gain. We will henceforth use the term Modulation Gain, denoted $G$, to represent the increase in capacity (bits/sec) using given modulation constellation relative to that of binary modulation. That is, $G$ is given by $G p=\log _{2}(M)$ where $M$ is the number of symbols in the modulation constellation. However, the penalty for such gain is an increase in BER and with it, an increase in the required number of retransmissions by the higher layer protocols (link layer ARQ or end-to-end ARQ). There is a clear trade-off between the block size of ARQ retransmissions will have a clear impact on the amount of redundant data that must be retransmitted on error, in which the block size of the ARQ plays an important role [7].

\section{THE MODEL}

The basic idea of the analytical model relies on assumptions of separation between Queuing Based Efficiency (QBE) and Error Based Efficiency (EBE) (see particularly [3] for a fuller explanation). The overall efficiency, or simply efficiency, is the ratio between the mean user bit rate generated by the customer and the required channel capacity: it is the product of the QBE and the EBE. In this paper we focus on the effect of block size on EBE. For a discussion of QBE, the reader is referred to [2], which shows how the values of QBE were obtained, and for a discussion of EBE optimisation not taking into account the effect of block size the reader is referred to [3].

Briefly, the EBE is the ratio between the mean user data rate and the user data rate plus all overheads associated with error corrections and retransmission of erroneous blocks of data, namely, FEC, and ARQ and adjusted for the Modulation Gain. Accordingly the EBE is a product of (1) the FEC code rate $R$ defined by the ratio: $R=$ (user data) / (user data + FEC redundancy); (2) the ARQ efficiency denoted $A$ and defined by the ratio: $A=$ (user data + FEC redundancy) / (user data + FEC redundancy + additional 
capacity for ARQ retransmissions ); and (3) the above defined Modulation Gain $(G)$.

In [3] an analytical formula for the Optimal $E B E$ denoted $E B E^{*}$ and defined as the maximal value of EBE subject to meeting QoS requirements was derived for an adaptive wireless multi-service system. We give the formula here for use in this paper but we refer the reader to [3] for derivation. The equation optimises values of $G$ and $R$ for a given ARQ block size and a particular FEC scheme. If $N$ is the ARQ block size in bits, then the probability that an ARQ block will be transmitted successfully is denoted by $\alpha$ and is given by:

$$
\alpha=(1-e(G, R))^{N}
$$

Given that $\alpha$ is a function of $G$ and $R$ the EBE is given by and and the utilization of the adaptive system is optimised by:

$$
E B E^{*}=\max _{G, R}[G \times R \times \alpha]
$$

We must optimize $G$ and $R$, considering the trade-off between the FEC redundancy and Modulation Gain versus the volume of retransmission of erroneous blocks of data (which will be affected by the blocksize, $N$ ). We conservatively assume independent bit errors.

Notice that the above does not consider packet delay due to ARQ retransmission. Nevertheless, in most wireless or mobile systems the propagation delay over the radio link is insignificant and hence a large number of retransmissions are possible without violating delay QoS requirements. However, when the radio link is long, eg for a satellite, or when ARQ is performed end to end at the TCP level over large distances, a constraint on the delay must be added to the optimisation of Eq. (2).

\subsection{Considering Block size, $N$}

From Eq.(1), it is clear as that BER increases, there will be a rapid fall in $\alpha$ and thus a fall in efficiency for given $G$ and $R$ as $e(G, R)$ for the fixed $G$ and $R$ becomes significant (see Eq. (2)). A larger $N$ will make this decline occur at a higher SNR (or $e(G, R)$ ) on the link, and increase the rate of decline of EBE against SNR than for a smaller $N$. In the non-adaptive system, this decline is not correctable and leads to the much discussed TCP 'slow start' problem [5]. That problem identified by authors concerning ATM over wireless links is that the window protocol mechanisms built into TCP are designed to avoid network congestion and do not take account of characteristics of the wireless environment (which was not a consideration in the design of TCP). Hence cell loss on any link of the network - which TCP assumes is wired, having low BER - must be as the result of network congestion rather than an errored packet. 
One solution, considered in [9] suggests the implementation of an additional small block ARQ in a specific Wireless Data Link Control Layer (rather than end-to-end ARQ) to deliver a better quality channel over the wireless link to the higher layer protocols. This implementation of small blocks at the data link layer provides error free transmission to higher layers thus avoiding inefficiencies in retransmission of TCP larger blocks. In this paper, we find that in our adaptive modulation model, such a scheme may not be necessary.

In the case where $N$ is variable, as for TCP over the wireless link, by Eq. (2), it is possible to obtain a condition for when $G$ and $R$ need to be changed from their origional values, $G_{l}$ and $R_{l}$ to new optimal values as a result of a change of $N$ from $N(1)$ to $N(2)$.

$$
G_{1} \times R_{1} \times\left(1-e\left(G_{1}, R_{1}\right)\right)^{N(1)}<\max _{G, R}\left[G \times R \times \alpha_{N(2)}\right]
$$

Eq. (3) gives this condition for a change of $G$ or $R$ in the adaptive system. Where the value of $N$ is variable, an increase in the value of $N$ will lead to a reassessment of optimal values of $G$ and $R$. In the case of a fixed modulation scheme, the optimization is only available over $R$ or not at all meaning that EBE will decline rapidly as SNR increases, leading to a smaller $N$ imposed by TCP/IP and a large loss in user utilisation. The adaptive scheme on the other hand is able to alter $R$ and $G$ to maintain service levels.

Since there is no analytical solution for the BER for a given set of $G$ and $R$, we have to rely on simulations where we commence with the optimisation of Modulation Gain and FEC and then consider blocksize $N$ for the optimized adaptive system. The details of the simulations are discussed in [3].

\section{THE SIMULATION}

Simulations used in this paper were developed using the Matlab Communications Toolbox [6]. The wireless environment was simulated using a Varying Rayleigh Fading Channel (VRFC) in which the parameters of the Rayleigh Fading Channel were varied randomly with uniform distribution. The simulation uses Minimum Phase Shift Keying (MPSK) which was preferred for simplicity, however the analysis presented is equally relevant for High Level Modulation including Enhanced Data rates for GSM Evolution (EDGE) [4] which uses Quaternary Offset QAM (Q-O-QAM). This approximation is justified since the analysis is independent of modulation scheme and the preferred modulation scheme for future wireless (or WATM) networks has not yet emerged. 


\section{RESULTS}

In this section we present numerical results displaying the relative insensitivity of our scheme to block size of the transmission, effectively providing a solution to concerns regarding the performance of TCP over WATM and other wireless multi-media systems.

Since Go Back $N$ requires end-to-end (not just wireless link) retransmission of all subsequent packets to the errored packet, and there is a sliding window, larger window size is equivalent to the retransmission of larger ARQ block sizes. Figure 1 demonstrates this argument. In this case we consider EBE with varying window size. As $S N R$ decreases and $e(R, G)$ increases, the protocol reduces TCP window size, and hence throughput.

Figure 1 shows firstly the EBE gain in lower SNRs for the fixed modulation case achieved as a result of reduction of window size from 53000 bytes to 530 bytes. It is clear that the utilisation curve for the non-adaptive case with smaller window goes rapidly to zero at a lower SNR, showing that a reduction in window size gives an increased $\mathrm{EBE}$ in cases of higher $e(R, G)$. Thus, although TCP reduces the window size assuming network congestion, it is also carrying out the correct EBE action for wireless cell loss. We note that our maximum window size is large, however, for an inter-continental link, this is a realistic size window for a bit rate of $20 \mathrm{Mbs}$.

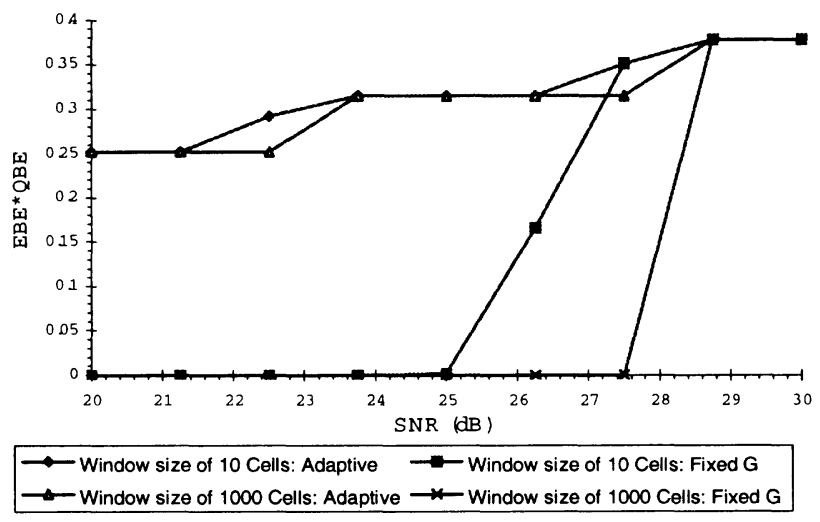

Figure 1. Adaptive and fixed modulation for uncoded MPSK system with ARQ. TCP window size equal to 530 and 53000 bytes. The fixed case is $G=6$. Overall Transmission Efficiency (EBE and QBE) is shown.

Secondly, Figure 1 shows that, for the case of adaptive modulation, the action of the transmitter is to move from one constellation to another when error becomes significant using the rule in Eq. (3). In this case, delay increases as the system comes closer to a transition point, since error and 
hence retransmission increases at this point. TCP decreases window size until the transmitter chooses a modulation constellation with greater redundancy, lower efficiency and consequential lower packet error.

The TCP window can then increase in response to the new conditions. Hence, TCP acts to 'cushion' the transfer between MEs. Note that as the TCP window size becomes small, there is a larger delay penalty in the overall utilisation that is not captured by the EBE (especially over long end-to-end distances), however when this becomes significant, the EBE in the larger window size is likely to be so poor as to render the smaller window size nonetheless more efficient.

In the case, as suggested in [7] that a data link layer small block ARQ is used exclusively on the wireless link in order to make the wireless link appear to the higher TCP/IP layer as if it is error free, Figure 2 compared with Figure 3 show that the adaptive system is relatively insensitive to blocksize (compared with the fixed system in Figure 1. Hence another advantage of the adaptive scheme is that any block size ARQ can be used in the wireless ARQ to provide good efficiency.

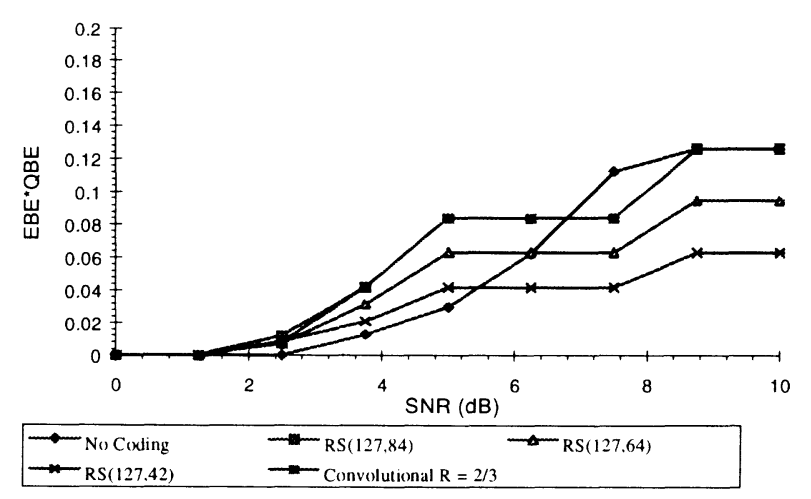

Figure 2. Efficiency comparison amongst several coding schemes; $A R Q$ block size $=$ one ATM cell ( 53 bytes) at low SNRs.

In [3] we find that optimal EBE is given by the adaptive modulation system in the absence of coding for most SNRs. In Figure 1 this is the case - changes to the system are made exclusively to $G$ and the maximum efficiency is given in the uncoded case. 


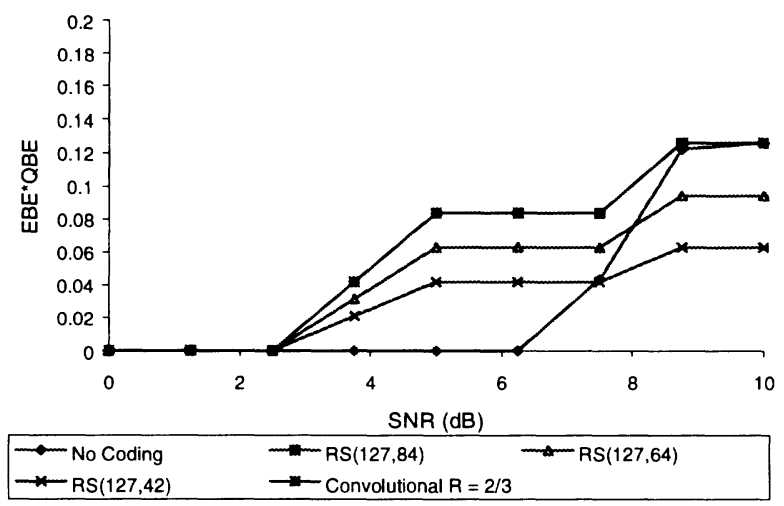

Figure 3. Efficiency comparison amongst coding schemes - ARQ block size $=100$ cells $(5300$ bytes) at low SNRs.

In Figures 2 and 3 we show that for very low SNR values, changes in coding are required to achieve optimal performance, particularly for larger block sizes. We note however that, even in this situation, the higher rate codes still give better performance than the more powerful codes with greater redundancy.

\section{CONCLUSION}

This paper considers the tradeoff of providing QoS for WATM at both the physical (modulation) and data link level (ARQ and FEC). It also considers the effect of the introduction of lower level ARQ (AAL). We show that the use of FEC with associated delay and complexity constraints can be avoided without violating the QoS bound. Our results show that that adaptive modulation offers significant efficiency and coverage gains over the fixed system when ARQ is considered and that in many instances an adaptive modulation system renders FEC redundant. We leave the implementation of the adaptive change of modulation parameters for another paper, however our findings suggest that preference should be given to systems that adaptively change their parameters according to the instantaneous level of interference and noise. We further observe that (1) the TCP assumption of a wired network, and the 'slow start' problem in particular, may not be a difficulty in wireless networks (2) that reduction in TCP window size has a beneficial effect in wireless transmission and (3) that even for certain realistic SNR conditions, the wastage due to errors can be very high because of retransmission. 


\section{References}

[1] H. ArmbrÜster, "The Flexibility of ATM: Supporting Future Multimedia and Mobile Communications," IEEE Personal Comm., pp.76-84, Feb. 1995.

[2] F. Cameron, M. Zukerman and M. Gitlits, "Wireless Link Dimensioning and Transmission Parameters Optimization," in K. Leung and B. Vojcic (eds.) Multiaccess, Mobility and Teletraffic for Wireless Communications: Volume 3, pp. 295-308, Kluwer Academic Publishers, Boston, 1999.

[3] F. Cameron, M. Zukerman, and M. Gitlits, "Adaptive Transmission Parameters Optimisation in Wireless Multi-access Communication", ICON '99 (Forthcoming)

[4] Ericsson, "EDGE Feasibility Study Work Item 184; Improved Data Rates through Optimised Modulation (Preliminary version 0.1)", SMG \#22, Munich, Germany, 12-16 May 1997.

[5] J. Cain and D. McGregor, "A recommended error control architecture for ATM networks with wireless links", (1997) 15(1) IEEE J Select Areas Comm 16.

[6] The Math Works Inc., Communications Tool Box for use with MATLAB and SIMULINK, April 1996.

[7] D. Raychaudhuri et al., "WATMnet: a prototype wireless ATM system for multimedia personal communication," IEEE J. Select. Areas Commun. vol. 15, no. 1, pp. 83-94, January 1997.

[8] M. Schwartz, "Network Management and Control Issues in Multimedia Wireless Networks," IEEE Personal Comm., pp. 8-16, June 1995.

[9] D. Turina, "Performance Evaluation of a Single-Slot Packet Data Channel in GSM", IEEE VTC, pp544-548, 1997

[10] M. Zukerman, P. L. Hiew and M. Gitlits, "FEC code rate and bandwidth optimisation in WATM networks", in D. Everitt and M. Rumsewicz (eds.), Multiaccess, Mobility and Teletraffic: Advances in Wireless Networks, Kluwer, Boston, 1998.

[11] M. Zukerman, P. L. Hiew and M. Gitlits, "Teletraffic Implications of a Generic ATM Wireless Access Protocol”, IEEE Globecom '97. 